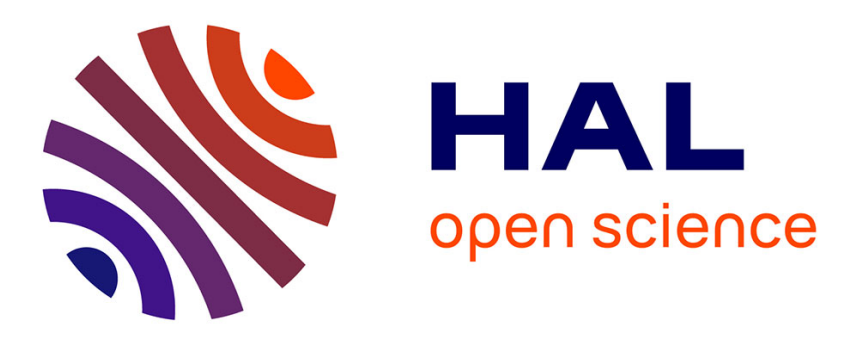

\title{
A Heavy Traffic Approach for Queue-Aware Power Control in Interfering Wireless Links
}

\author{
Apostolos Destounis, Mohamad Assaad, Mérouane Debbah, Bessem Sayadi, \\ Afef Feki
}

\section{- To cite this version:}

Apostolos Destounis, Mohamad Assaad, Mérouane Debbah, Bessem Sayadi, Afef Feki. A Heavy Traffic Approach for Queue-Aware Power Control in Interfering Wireless Links. 2012 IEEE 13th International Workshop on Signal Processing Advances in Wireless Communications (SPAWC), Jun 2012, Izmir, Turkey. pp.184 - 188, 10.1109/SPAWC.2012.6292884 . hal-00770636

\section{HAL Id: hal-00770636 \\ https://hal-centralesupelec.archives-ouvertes.fr/hal-00770636}

Submitted on 8 Jan 2013

HAL is a multi-disciplinary open access archive for the deposit and dissemination of scientific research documents, whether they are published or not. The documents may come from teaching and research institutions in France or abroad, or from public or private research centers.
L'archive ouverte pluridisciplinaire HAL, est destinée au dépôt et à la diffusion de documents scientifiques de niveau recherche, publiés ou non, émanant des établissements d'enseignement et de recherche français ou étrangers, des laboratoires publics ou privés. 


\title{
A HEAVY TRAFFIC APPROACH FOR QUEUE-AWARE POWER CONTROL IN INTERFERING WIRELESS LINKS
}

\author{
Apostolos Destounis ${ }^{\star}$, Mohamad Assaad ${ }^{\dagger}$, Mérouane Debbah ${ }^{\dagger}$,Bessem Sayadi ${ }^{\star}$, Afef Feki ${ }^{\star}$ \\ * Alcatel-Lucent Bell Labs France, Route de Villejust, 91620, Nozay, cedex. France \\ ${ }^{\dagger}$ SUPÉLEC, 3 rue Joliot-Curie, 91192, Gif-sur-Yvette, cedex. France
}

\begin{abstract}
In this work, we address the problem of power allocation for interfering transmitter-receiver pairs so that the probability that each queue length exceeds a specified threshold is fixed at a desired value. One application is satisfying QoS requirements in a dense cellular network. We address this problem using heavy traffic approximation techniques which lead to an asymptotic model described by a (controlled) stochastic differential equation. The power control strategy consists in allocating most of the power according to the wireless channel state and a smaller fraction according to the queue lengths. Simulation results in a simple setting illustrate that the proposed control policy can yield desirable results in practical systems.
\end{abstract}

\section{INTRODUCTION}

The explosive growth in the demands of mobile users, mainly due to video and data applications being increasingly popular on mobile phones, poses a serious challenge to cellular network operators. A promising way to deal with this demand is the concept of Small Cell Networks (SCN), which are dense cellular networks with low power base stations and frequency reuse one [1]. One of the key issues arising in these networks is that the intercell interference becomes severe. Cooperation between the base stations of a cellular network has been shown to mitigate the intercell interference and increase spectral efficiency [2]. However, this is not always feasible due to practical limitations in the backhaul capacity. Moreover, in these works incoming traffic pattern is not taken into account. Therefore a better approach for practical cases is to make the resource allocation at the base stations taking into account the real-time traffic characteristics and queue states.

Regarding the problem of simultaneous transmissions over interfering channels, substantial work has been done in power allocation so that the SINR at each receiver is above a specified threshold [3]. This approach however is not suited for the traffic nature of data and video streaming applications, as the resulting power control algorithms do not adapt to the traffic, queue states and/or the specific requirements of the application requested. Closer to this direction, in [4] a scheduler based on $H$-infinity control was proposed in order to regulate the buffers of small cell base stations around a target length.
The authors in [5] address the problem of minimizing a global function of the queue lengths that corresponds to the expectation of the sum of the delays in each base station. Therein, an algorithm that uses dynamic scheduling (inside the cell) and power allocation (for intercell interference mitigation) is proposed.

In this paper we consider a system of $K$ transmitter-receiver pairs. Our goal is to set the probability that the queue length at each transmitter exceeds a specified threshold at a desired value, i.e.

$$
\operatorname{Pr}\left\{q_{k}(t)>q_{k}^{t h r}\right\}=\delta_{k}, \forall k=1, \ldots, K .
$$

These constraints can correspond to fixing the data loss ratio due to buffer overflow or regulating the delay for each user. The setting of the interfering pairs can correspond to small cells employing the same subcarrier(s) to serve the users.

The approach followed in this paper is based on the heavy traffic asymptotic modeling [6]. In this method, the network is examined for the case when the offered traffic is almost equal to the mean service rate. In this regime, the system model becomes more tractable to study analytically and obtain control strategies. Our work is based on [7] and [8], where this method was used for power control in a point-to-point link and a single cell multiuser system respectively. However, they do not consider neither restrictive constraint as in (1) nor interference. We extend therefore these works in interfering channels, where the main issue is that the power allocation at each transmitter affects all the others. The general idea is that power is allocated first according to the channels so that the average rate is equal to the average incoming traffic rate and a small reserve amount is added for the system to adapt to the instantaneous traffic dynamics. The reason behind this reserve power allocation is that when the average rate equals the average input rate, the delay becomes unbounded [9]. We consider a centralized approach with full knowledge of all the channels and the queue lengths at each transmitter. The contributions of this paper are: $(i)$ the heavy traffic modeling of power control in interference channels and $(i i)$ the derivation of a method to satisfy (1) for all users under this centralized assumption.

The rest of this paper is organized as follows: In Sec. 2 we derive the heavy traffic model as a controlled Stochas- 
tic Differential Equation which we use in Sec.3 to devise a power control algorithm so that (1) is satisfied. Finally, Sec. 4 presents the corresponding simulation results and Sec. 5 concludes the paper.

\section{HEAVY TRAFFIC SYSTEM MODEL}

We consider a system of $K$ transmitters each serving one receiver using bandwidth $W$, as illustrated in Fig. 1. For notational simplicity, we index the transmitters and the receivers so that transmitter $k$ serves user $k$. Also, let $g_{i j}(t)$ denote the power gain of the channel between transmitter $i$ and user $j$ at time $t$. Each of these channel gains is assumed to evolve independently of the others as an ergodic finite state Markov chain [10]. Under this assumption then, the matrix $\mathbf{G}(t)=\left[g_{i j}(t)\right]$ of all channel gains at time $t$ will also evolve as an ergodic finite state Markov chain with, say, $M_{G}$ possible states . Let us define the set of possible channel realizations as $S_{G}=\left\{\mathbf{G}_{\mathbf{1}}, \ldots, \mathbf{G}_{M_{G}}\right\}$. We shall denote the event that the channel gain matrix is in the $m$-th state as $\mathbf{G}(t)=\mathbf{G}_{m}$. The corresponding ergodic probability will then be denoted as $\pi_{m}$, and $\mathbb{E}_{\pi}\{\}$ denotes the expectation over this probability distribution. When transmitter $k$ uses power $p_{k}(t)$, the rate $r_{k}(\mathbf{p}(t), \mathbf{G}(t))$ over the link with its corresponding receiver will be assumed as the Shannon rate treating interference as Gaussian noise, that is

$r_{k}(\mathbf{p}(t), \mathbf{G}(t))=W \log _{2}\left(1+\frac{g_{k k}(t) p_{k}(t)}{\sum_{i=1, i \neq k}^{K} g_{i k}(t) p_{i}(t)+\sigma^{2}}\right)$,

where $\sigma^{2}$ is the noise variance and $\mathbf{p}(t)$ the vector of transmission powers.

For each queue we suppose that the instantaneous arrivals $a_{k}(t)$ at time $t$ are i.i.d. with mean $\lambda_{k}$ and (finite) variance $\sigma_{a, k}^{2}$ and independent of the arrivals at the other queues and the channel process.

We will work in the Heavy Traffic regime [6], which intuitively means that the average transmission rate at each transmitter will be almost equal to the mean rate of the incoming traffic. Formally, as it is fairly standard in the literature [6], time and magnitudes are scaled by $n$ and the system in the limit as $n \rightarrow \infty$, derived from some form of central limit theorem, is examined. The interpretation of the parameter $n$ can be as the order of magnitude of the arrivals; i.e. at any time interval $\Delta t$ there are $O(n \Delta t)$ arrivals. The limit implies that in the heavy traffic situations there are too many arrivals in the transmitters and they are almost never idle. Let $a_{k}^{(n)}(t)$ denote the arrival process at transmitter $k$ at the $n$-th system. Then, for transmitter $k$ we assume that $a_{k}^{(n)}(t) \rightarrow a_{k}(t)$, where $a_{k}^{(n)}(t)$ have mean rate $\lambda_{k}^{(n)} \rightarrow \lambda_{k}$ and $\sigma_{a, k}^{(n)} \rightarrow \sigma_{a, k}$. Also, denote $\mathbf{p}^{(n)}(t)$ the power allocation vector at time $t$ for the $n$-th system.

Following [7] and [8], we also parametrize the number of channel changes with the integer $n$ and assume that the channels change also fast but at a slower rate than the incoming traffic. Thus, at the time interval $\Delta t$ there will be $O\left(n^{\nu} \Delta t\right)$ channel changes, for a $0<\nu<1$. In other words, the exponent $\nu$ is selected such that $n^{\nu}$ is the order of the rate with which the channels change. Now, let $q_{k}(t)$ denote the queue length of transmitter $k$ at time $t$, and $x_{k}^{(n)}(t)$ the scaled version as follows:

$$
x_{k}^{(n)}(t)=\frac{1}{n^{\frac{\nu}{2}}} q_{k}\left(n^{\nu} t\right),
$$

that is the time and queue lengths are scaled based on the rate at which the environment (channels) changes. The use of central limit theorems as $n$ goes to infinity implies thus an averaging of the system's behaviour over many channel changes. The power allocation corresponding to the $n$-th system is

$$
p_{k}^{(n)}(t)=\bar{p}_{k}\left(\mathbf{G}^{(n)}(t)\right)+\frac{1}{n^{\frac{\nu}{2}}} u_{k}\left(\mathbf{x}^{(n)}(t), \mathbf{G}^{(n)}(t)\right),
$$

where $\bar{p}_{k}\left(\mathbf{G}^{(n)}(t)\right)$, called "equilibrium allocation" for the rest of this paper, satisfies

$$
\lambda_{k}=\mathbb{E}_{\pi}\left\{r_{k}(\overline{\mathbf{p}}(\mathbf{G}(t)))\right\}, \forall k=1, \ldots, K .
$$

Also, $u_{k}$ is a function of the queue lengths and channel states and corresponds to the reserve power allocation around the equilibrium. Using (4) and a Taylor expansion of (2), the rate at each transmitter for the sequence of systems can be described as

$r_{k}^{(n)}(t)=r_{k}\left(\overline{\mathbf{p}}\left(\mathbf{G}_{m}\right)\right)+\frac{1}{n^{\frac{\nu}{2}}} \sum_{i=1}^{K} a_{k, i}\left(\mathbf{G}_{m}\right) u_{i}\left(\mathbf{x}(t), \mathbf{G}_{m}\right)+o\left(n^{-\frac{\nu}{2}}\right)$,

where $a_{k, i}\left(\mathbf{G}_{m}\right)=\frac{\partial r_{k}\left(\overline{\mathbf{p}}\left(\mathbf{G}_{m}\right)\right)}{\partial p_{i}}$ Moreover, the scaled queue length (3) at each transmitter $k$ is

$$
\begin{aligned}
x_{k}^{(n)}(t)= & x_{k}^{(n)}(0)+\frac{1}{n^{\nu / 2}} \int_{0}^{n^{\nu} t} a_{k}^{(n)}(s) d s \\
& +\frac{1}{n^{\nu / 2}} \int_{0}^{n^{\nu} t} r_{k}^{(n)}(s) d s+z_{k}^{(n)}(t),
\end{aligned}
$$

where $z_{k}^{(n)}(t)$ is such that the queue length process is nowhere negative. Intuitively, it represents a "wasted" service until time $n^{\nu} t$, i.e. the number of bits that would have been transmitted in the periods where the queue was empty.

As $n \rightarrow \infty$ we can show, using the techniques in [7] and [8], that the vector of the scaled queue length processes converges weakly to $\mathbf{x}(t)=\left[x_{1}(t), \ldots, x_{K}(t)\right]^{T}$, given as

$$
\mathbf{x}(t)=\mathbf{x}(0)-\int_{0}^{t} \mathbf{f}(\mathbf{u}(s)) d s+\mathbf{\Sigma} \mathbf{w}(t)+\mathbf{z}(t) .
$$

In the above, $\mathbf{w}(t)$ is a vector of $K$ independent standard Wiener processes, $\mathbf{f}$ is the vector of the functions

$$
f_{k}(\mathbf{u}(t))=\sum_{m=1}^{M_{G}} \pi_{m} \sum_{j=1}^{K} a_{k, j}\left(\mathbf{G}_{m}\right) u_{j}(t)
$$

and the matrix $\boldsymbol{\Sigma}=\left[\sigma_{i j}\right]$ satisfies

$$
\boldsymbol{\Sigma} \boldsymbol{\Sigma}^{T}=\boldsymbol{\Sigma}_{a} \boldsymbol{\Sigma}_{a}^{T}+\boldsymbol{\Sigma}_{d} \boldsymbol{\Sigma}_{d}^{T}
$$


In the above equation, $\boldsymbol{\Sigma}_{a}=\operatorname{diag}\left(\sigma_{a, k}\right)$ while the elements of the covariance matrix $\boldsymbol{\Sigma}_{d} \boldsymbol{\Sigma}_{d}^{T}=\left[s_{i j}\right]$ are given as [8]

$$
s_{i j}=2 \mathbb{E}\left\{\int_{0}^{+\infty} \hat{r}_{i}(0) \hat{r}_{j}(t) d t\right\}
$$

where $\hat{r}_{k}(t)=r_{k}(\overline{\mathbf{p}}(\mathbf{G}(t)))-\lambda_{k}$. Note that $\boldsymbol{\Sigma}_{d}$ is not diagonal since the equilibrium rates depend on the same stochastic process, the matrix of the channel gains in our case.

Finally, the elements of $\mathbf{z}(t)$ are given as

$$
\begin{aligned}
& z_{k}(t)= \\
& {\left[-\min _{s \leq t}\left\{x_{k}(0)-\int_{0}^{t} f_{k}(\mathbf{u}(s)) d s+\sum_{j=1}^{K} \sigma_{k j} w_{j}(t)\right\}\right]^{+},}
\end{aligned}
$$

where $[x]^{+}=\max \{0, x\}$, so that $x_{k}(t) \geq 0$. If there was routing of incoming data from one transmitter to another, in the equation there would be this process multiplied by a corresponding matrix (reflection matrix [6]); since here this is not the case, this reflection matrix is the unitary.

Note also that (9) implies that the drift in (8) is actually the expected value, under the ergodic probability distribution of the channel gain matrix, of the effect of the fluctuations $\mathbf{u}(t)$ of the power around the equilibrium allocation. Therefore, the heavy traffic model can be seen as an averaged model over the random environment.

\section{PROPOSED POWER CONTROL POLICY}

\subsection{Equilibrium Power Allocation}

As we can see from (4), the resource allocation policy consists of two parts: $(i)$ determining the equilibrium power allocation according to the state of the channels and (ii) determining the reserve power allocation according to the channels and queue lengths. In this work, we set the equilibrium power allocation such that the rates at each possible state of the channels are equal to the mean rates of the incoming traffic (assuming this is feasible). This corresponds to each link having the same SINR at the receiver for each state of the channel gain matrix, so the equilibrium power allocation can be computed solving a system of linear equations for each $\mathbf{G}_{m}$ [3]. Under this equilibrium allocation, $\boldsymbol{\Sigma}_{d}$ is a zero-element matrix, since there is no variation of the rate in the equilibrium allocation for different channel states. We also set the reserve power allocation to depend only on the queue lengths, i.e. $\mathbf{u}(t)=\mathbf{u}(\mathbf{x}(t))$. The intuition behind this feedback control form is that the goal of the reserve power allocation is to actually regulate the queue lengths while the equilibrium power allocation deals with the channel states. In this case then, we can write (9) in the following form (with the differentials being in the Itô sense):

$$
d \mathbf{x}(t)=\mathbf{B u}(\mathbf{x}(t)) d t+\mathbf{\Sigma} d \mathbf{w}(t)+d \mathbf{z}(t) .
$$

In the above equation $\boldsymbol{\Sigma}=\operatorname{diag}\left(\sigma_{a, k}\right)$ and $\mathbf{B}=\left[b_{i j}\right]$ with $b_{i j}=-\sum_{m=1}^{M_{G}} a_{i, j}\left(\mathbf{G}_{m}\right) \pi_{m}$.

\subsection{Reserve Power Allocation}

We now focus on the reserve power allocation, namely determining the fluctuation around the equilibrium powers in order to regulate the queue lengths.

Proposition 1. With the equilibrium allocation of the previous section, the overflow requirements for the asymptotic system can be satisfied by the following policy:

$$
\mathbf{u}(\mathbf{x}(t))=\mathbf{B}^{-1} \mathbf{C x}(t),
$$

with $\mathbf{C}=\operatorname{diag}\left(-\left|c_{k}\right|\right)$ and

$$
\left|c_{k}\right|=\frac{1}{2}\left(\frac{\sigma_{a, k}}{x_{k}^{t h r}} \operatorname{erfc} c^{-1}\left(\delta_{k}\right)\right)^{2} .
$$

By $\operatorname{erfc}(x)$ we denote the complementary error function, that is erfc $(x)=\frac{2}{\sqrt{\pi}} \int_{x}^{+\infty} e^{-t^{2}} d t$ and $x_{k}^{t h r}$ is the corresponding threshold in the asymptotic regime.

Proof. Let us first start by establishing the probabilistic framework of the stochastic differential equation in (13). Let now $\left(\Omega, \mathcal{F},\left\{\mathcal{F}_{t}\right\}_{t \geq 0}, \mathcal{P}\right)$ be a complete probability space satisfying the usual hypotheses, i.e., $\mathcal{F}_{o}$ contains all the $\mathcal{P}$-null sets of $\mathcal{F}$ and $\left\{\mathcal{F}_{t}\right\}_{t \geq 0}$ is a right continuous filtration of $\sigma$-algebras. The Wiener Process $\mathbf{w}(t)=\left(w_{1}(t), \ldots, w_{K}(t)\right)_{t \geq 0}^{T}$ is $\left\{\mathcal{F}_{t}\right\}_{t \geq 0^{-}}$ adapted with stationary and independent increments. This process is also independent of the initial state $\mathbf{x}_{0}$ which is an $\mathcal{F}_{o}$-measurable random variable with finite second moment. The reflection process $z(t)=\left(z_{1}(t) \ldots z_{K}(t)\right)_{t \geq 0}^{T}$ is a continuous non-decreasing $\left\{\mathcal{F}_{t}\right\}_{t \geq 0}$-adapted $\mathbb{R}_{+}^{K}$ valued process and each $z_{k}(t)$ increase only when $x_{k}(t)=0$. We define our control policy in the class of Markov feedback control (i.e. the control depends on $\mathbf{x}(t)$ ). It is well known that the existence of Markov control is related to the existence of solution for the corresponding SDE. Applying the proposed controller, the states are decoupled as

$$
d x_{k}(t)=-\left|c_{k}\right| x_{k}(t) d t+\sigma_{a, k} d w_{k}(t)+d z_{k}(t) .
$$

Our control policy makes the evolution of the queues decoupled which is very useful to ensure the existence of a solution to the SDE. Since the states are decoupled and using the reflection direction in (12), we can show that the controlled process $\mathbf{x}(t)$ is positive recurrent. Using the main result of [11], the controlled process in (16) has a unique invariant probability measure which is absolutely continuous with respect to the Lebesgue measure, i.e. has a density that can be obtained using the Fokker-Planck equation as $\phi\left(x_{k}\right)=$ $\sqrt{\frac{\left|c_{k}\right|}{\pi \sigma_{a, k}^{2}}} e^{-\left|c_{k}\right| x_{k}^{2} / \sigma_{a, k}^{2}}$. According to the ergodic properties of recurrent diffusion processes, we can use the above density of the invariant measure to compute the overflow of the controlled stochastic process $\mathrm{x}(\mathrm{t})$

$$
\operatorname{Pr}\left\{x_{k}(t)>x_{k}^{t h r}\right\}=\operatorname{erfc}\left(\frac{x_{k}^{t h r} \sqrt{2\left|c_{k}\right|}}{\sigma_{a, k}}\right) .
$$


Replacing the overflow probability with its desired value and solving (17) we get (15), which completes the proof.

Note that the control policy regarding the reserve power is a closed form expression of the queue lengths. Given that the equilibrium power allocation is precomputed and under the assumption of complete queue and CSI knowledge this implies that at each time the control policy can be implemented in one shot instead of having an iterative algorithm (as it is done e.g. in [5]).

\subsection{Practical Implementation}

So far we worked on continuous time models whereas a practical communications system the time is slotted so power allocation decisions are taken into discrete time instances. More specifically, let the duration of each timeslot of the "real" (unscaled) system be $T_{s}$; this implies that at the $n$-th system of the sequence the timeslot duration is $T_{s}^{(n)}=T_{s} n^{-\nu}$. Moreover, the derivation of the control policy was concerned with an asymptotic system model, that is a model as a parameter $n$ goes to infinity. However, the control policy presented in the previous section has to be modified in order to operate on a real system, so the results obtained for the asymptotic case have to be converted into results for the unscaled system. This is done using (3) and (4), where the scaling parameter $n$ is now a big finite number. By definition, at a time interval $\delta t$ there are $O(n \delta t)$ arrivals, hence we can argue that in practical cases $n$ is in the same order of magnitude as average bit rates in the system. As far as the exponent $\nu$ is concerned, it can be obtained using the fact that during the coherence time, $T_{c o h}$, there must be only one channel change, thus $n^{\nu} T_{\text {coh }}=1$. Finally, the timeslot duration is chosen to be smaller than the coherence time, so that the channels can be considered static within one timeslot.

Based on the previous analysis, at the system where the queue lengths are $\mathbf{q}\left(l T_{s}\right)$ at the beginning of timeslot $l$, the power allocated for the duration of this timeslot will become (applying time slotting and unscaling using (3))

$$
\mathbf{p}\left(l T_{s}\right)=\overline{\mathbf{p}}\left(\mathbf{G}\left(l T_{s}\right)\right)+\mathbf{B}^{-1} \mathbf{C}^{\prime} \mathbf{q}\left(l T_{s}\right),
$$

where $\mathbf{C}^{\prime}=\operatorname{diag}\left(\left|c_{k}^{\prime}\right|\right)$ is obtained by (15) replacing the scaled queue length threshold with its unscaled value, $q_{k}^{t h r}$. Note also that since in (17) both quantities inside the probability are scaled ones, this is also the probability in (1), that is, the original objective.

Consider now the dynamics of the asymptotic system in discrete time; unscaling the quantities in (16) we get that the queue lengths evolve along timeslots approximately as

$$
q_{k}\left((l+1) T_{s}\right)=\left[\left(1-\left|c_{k}^{\prime}\right| T_{s}\right) q_{k}\left(l T_{s}\right)+T_{s} \sigma_{a, k} n_{k}\left(l T_{s}\right)\right]^{+}
$$

where $n_{k}\left(l T_{s}\right)$ is a discrete time AWGN with unitary variance. For the above not to diverge there must hold the condition $\left\|1-\left|c_{k}^{\prime}\right| T_{s}\right\|<1$ thus

$$
q_{k}^{t h r} \geq \frac{\sigma_{a, k}}{2} \sqrt{T_{s}} \operatorname{erfc} c^{-1}\left(\delta_{k}\right) .
$$

The above gives a lower bound on the thresholds that can be exceeded with a given probability using our control policy.

\section{SIMULATION RESULTS}

In order to illustrate the results of the power control method proposed in this paper, let us consider a simple scenario with 3 interfering transmitter - receiver pairs, using the same spectrum with bandwidth $5 \mathrm{MHz}$. For simplicity, we consider that each channel gain has only two possible values. Also, the arrivals at each transmitter are set as Poisson processes with mean rates $1,1.5$ and $2 \mathrm{Mbps}$. The overflow thresholds are set to $500,750,1000$ bits at each transmitter respectively and the overflow probability to 0.01 for all transmitters. The coherence time of the channels is set to $20 \mathrm{~ms}$, modelling slow fading channels, for instance in indoor or low mobility environments. The timeslot duration is set to $2 \mathrm{~ms}$, as is the shortest frame duration in HSDPA.

For this setting, we found that all the equilibrium powers are in the order of magnitude of $m W$. The expected values of the equilibrium powers over the ergodic distribution of the channel gains matrix were found by simulations to be $33.1 \mathrm{~mW}, 41.1 \mathrm{~mW}, 45.9 \mathrm{~mW}$ for transmitters 1,2 and 3 respectively. Also, for our proposed algorithm simulations showed that the reserve power allocated is in the order of magnitude of $10^{-5} \mathrm{~W}$, thus confirming the assumption of the reserve power being much smaller than the equilibrium power. Besides, we found that the average reserve powers used was $0.7 \times 10^{-5} W, 0.7 \times 10^{-5} W, 0,8 \times 10^{-5} W$ for each transmitter.

We compare our proposed method with the policy where all powers take their equilibrium values, and also with the following heuristic power allocation strategies: In the first one, the power at each transmitter is constant for every queue length and channel state and equal to the expectation of the equilibrium power over the ergodic distribution of the channel gain matrix plus the average reserve power presented above. The other heuristic strategy consists of adding the average reserve power to the equilibrium allocation. In the presentation of the results, the former is denoted as "Static power allocation" and the latter as "Channel-aware power allocation". These configurations were made to ensure that the amount of power available in the heuristic schemes is approximately the same as the power used in our proposed method, thus making a fair comparison. Due to space limitations we show only the results concerning the queue of one transmitter.

The performance of the aforementioned power allocation policies for each queue are shown in Fig. 2. We can see that equilibrium and static power allocations perform very bad. For the static power allocation case, the reason for this bad performance is the fact that the power does not take into account at all the channel states and the queue lengths. As far as the equilibrium power allocation is concerned, the bad performance is explained because the traffic and the queue lengths are not taken into account. Analyzing this policy further, as implied by (14) putting the vector of the reserve powers $\mathbf{u}$ 
equal to zero, the queue lengths behave like Wiener processes as confirmed also in our simulation setting in Fig. 3. In Fig. 2 it is clearly illustrated that assigning slightly more power in the equilibrium power for each channel state, as it is done in the channel-aware power allocation scheme defined earlier, leads to a much better performance, even if this extra power is really small. This was also expected as an increase in all the powers leads to an increase in the average rates. The equilibrium power allocation is exactly the point where the mean arrival rates equal the mean service rates thus even a small increase of the service rates is enough to stabilize the system. Achieving a better performance than the static power allocation case was also expected due to the transmission powers adapting to the channel conditions. Finally, we can observe that our proposed method of power allocation does even better, illustrating the additional advantage of taking the queue lengths into account when allocating the reserve power. Moreover, the overflow ratio is very close to the desired one, which implies the validity of the asymptotic model in a practical system operating under heavy load.

In addition, in Fig. 3 we can see the evolution of the queue length over time for a simulation run. We can observe that the queue length under the proposed power control method behaves in a much more controlled manner compared to the equilibrium power allocation and is below its respective threshold for most of the time, as suggested by our theoretical analysis.

\section{CONCLUSIONS}

In this paper we worked in the heavy traffic asymptotic regime in order to model the evolution of the queue lengths at the transmitters in a system of interfering wireless links and to propose a power allocation policy to keep the exceedence probabilities of the queue lengths in a desired value. Simulations showed that the closed form policy derived can give good results when applied in a practical system. Future work will address the problem in a decentralized setting.

\section{REFERENCES}

[1] J. Hoydis, M. Kobayashi, and M. Debbah, "Green small-cell networks," IEEE Veh. Technol. Mag., vol. 6, no. 1, pp. 37 -43, Mar. 2011.

[2] M. Karakayali, G. Foschini, and R. Valenzuela, "Network coordination for spectrally efficient communications in cellular systems," IEEE Trans. Wireless Commun., vol. 13, no. 4, pp. 56-61, Aug. 2006.

[3] M. Chiang, P. Hande, T. Lan, and C. W. Tan, "Power control in wireless cellular networks," Foundations and Trends in Networking, vol. 2, pp. 381-533, Apr. 2008.

[4] S. Lakshminarayana, M. Assaad, and M. Debbah, "H-infinity control based scheduler for the deployment of small cell networks," in WiOpt2011, May 2011, pp. 9-16.

[5] H. Huang and V. Lau, "Delay-optimal user scheduling and inter-cell interference management in cellular network via distributive stochastic learning," IEEE Trans. Wireless Commun., vol. 9, no. 12, pp. 3790 3797, Dec. 2010.

[6] H. J. Kushner, Heavy Traffic Analysis of Controlled Queuing and Communication Networks. Berlin and New York: Springrer-Verlag, 2001.

[7] W. Wu, A. Arapostathis, and S. Shakkottai, "Optimal power allocation for a time-varying wireless channel under heavy-traffic approximation," IEEE Trans. Autom. Control, vol. 51, no. 4, pp. 580 - 594, Apr. 2006.

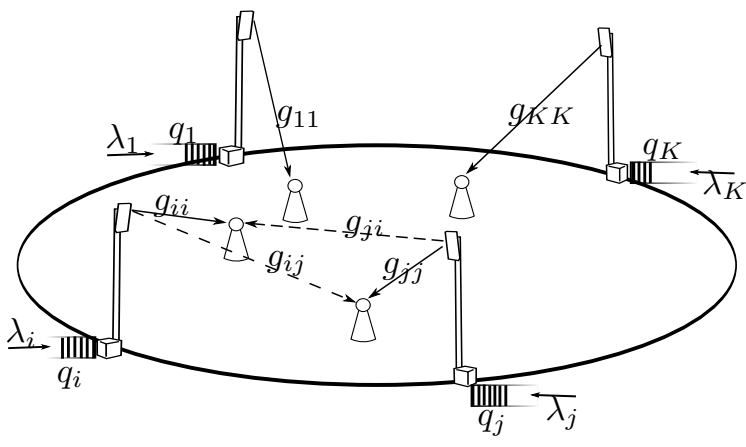

Fig. 1. Illustration of the system model

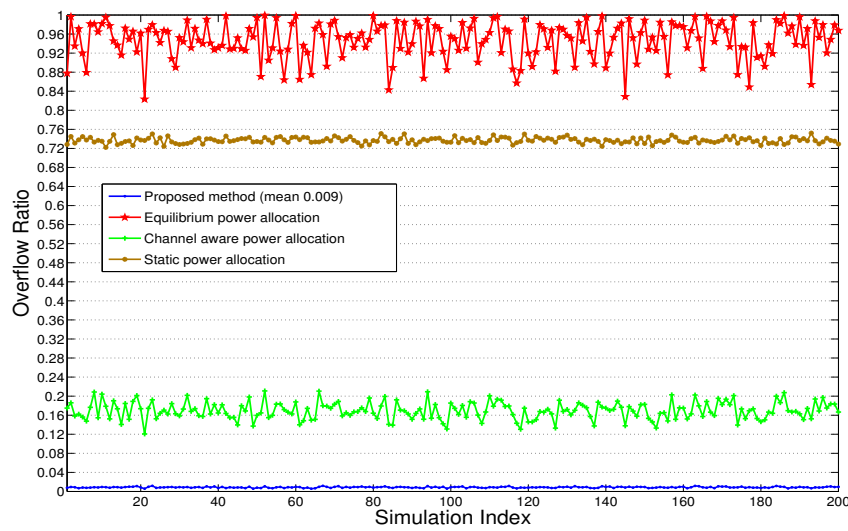

Fig. 2. Overflow ratios for the queue at link 1 . The target overflow ratio is 0.01 and the mean overflow ratio using the proposed method was 0.009 .

[8] R. Buche and H. J. Kushner, "Control of mobile communications with time-varying channels in heavy traffic," IEEE Trans. Autom. Control, vol. 47, no. 6, pp. $992-1003$, Jun. 2002.

[9] R. Berry and R. Gallager, "Communication over fading channels with delay constraints," IEEE Trans. Inf. Theory, vol. 48, no. 5, pp. 11351149, May 2002.

[10] H. S. Wang and N. Moayeri, "Finite-state markov channel-a useful model for radio communication channels," IEEE Trans. Veh. Technol., vol. 44, no. 1, pp. 163 -171, Feb. 1995.

[11] A. R. Ward and P. W. Glynn, "Properties of the Reflected Ornstein Uhlenbeck Process,," Queueing Systems, vol. 44, pp. 109-123, 2003.

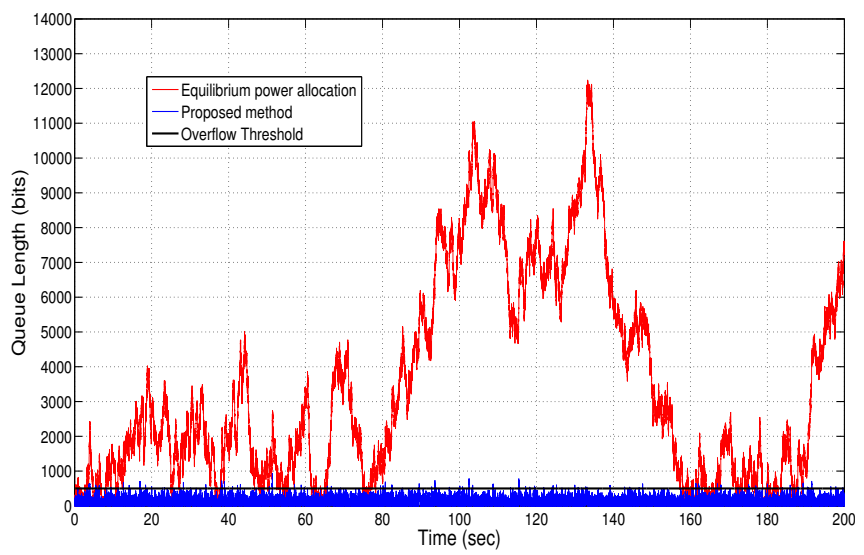

Fig. 3. Example of the queue length of link 1 evolution over time for the equilibrium allocation and the proposed policy. 